Geometry $\&$ Topology

Volume 8 (2004) 925-945

Published: 9 June 2004

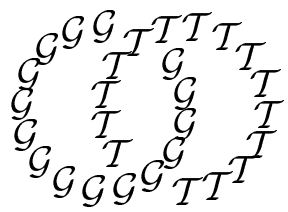

\title{
Ozsváth-Szabó invariants and tight contact three-manifolds, I
}

\author{
PaOlo Lisca \\ ANDRÁs I STIPSICZ \\ Dipartimento di Matematica, Università di Pisa \\ I-56127 Pisa, ITALY \\ and \\ Rényi Institute of Mathematics, Hungarian Academy of Sciences \\ H-1053 Budapest, Reáltanoda utca 13-15, Hungary \\ Email: lisca@dm.unipi.it and stipsicz@math-inst.hu
}

\begin{abstract}
Let $S_{r}^{3}(K)$ be the oriented 3-manifold obtained by rational $r$-surgery on a knot $K \subset S^{3}$. Using the contact Ozsváth-Szabó invariants we prove, for a class of knots $K$ containing all the algebraic knots, that $S_{r}^{3}(K)$ carries positive, tight contact structures for every $r \neq 2 g_{s}(K)-1$, where $g_{s}(K)$ is the slice genus of $K$. This implies, in particular, that the Brieskorn spheres $-\Sigma(2,3,4)$ and $-\Sigma(2,3,3)$ carry tight, positive contact structures. As an application of our main result we show that for each $m \in \mathbb{N}$ there exists a Seifert fibered rational homology 3-sphere $M_{m}$ carrying at least $m$ pairwise non-isomorphic tight, nonfillable contact structures.
\end{abstract}

\section{AMS Classification numbers Primary: 57R17}

Secondary: 57R57

Keywords: Tight, fillable contact structures, Ozsváth-Szabó invariants

Proposed: Peter Ozsváth

Seconded: John Morgan, Tomasz Mrowka
Received: 21 February 2004

Accepted: 29 May 2004 


\section{Introduction}

According to a classical result of Lutz and Martinet, every closed, oriented 3manifold admits a positive contact structure. In fact, every oriented 2-plane field on an oriented 3-manifold is homotopic to a positive contact structure. The proof of the Lutz-Martinet theorem - relying on contact surgery along transverse links in the standard contact 3-sphere [13 - typically produces overtwisted contact structures. (For a proof of the Lutz-Martinet theorem using contact surgery along Legendrian links see [6].) Finding tight contact structures on a closed 3-manifold is, in general, much more difficult, indeed impossible for the Poincaré homology 3-sphere with its natural orientation reversed [12].

Let $Y$ be a closed, oriented 3-manifold. Consider the following problem:

(P) Does $Y$ carry a positive, tight contact structure?

Until recently, the two most important methods to deal with problem $(\mathrm{P})$ were Eliashberg's Legendrian surgery as used eg by Gompf in [14, and the state traversal method, developed by Ko Honda and based on Giroux's theory of convex surfaces. The limitations of these two methods come from the fact that Legendrian surgery can only prove tightness of Stein fillable contact structures, while the state traversal becomes combinatorially unwieldy in the absence of suitable incompressible surfaces. For example, both methods fail to deal with problem $(\mathrm{P})$ when $Y$ is one of the Brieskorn spheres $-\Sigma(2,3,4)$ or $-\Sigma(2,3,3)$, because these Seifert fibered $3-$ manifolds do not contain vertical incompressible tori, nor do they carry symplectically fillable contact structures [18].

The purpose of the present paper is to show that contact Ozsváth-Szabó invariants 28, can be effectively combined with contact surgery [4, 5] to tackle problem (P). In particular, it follows from Theorem 1.1 below that $-\Sigma(2,3,4)$ and $-\Sigma(2,3,3)$ do indeed carry tight, positive contact structures. Moreover, such contact structures admit an explicit description (cf Corollary 1.2 and the following remark).

In order to state our main result we need to introduce some notation. Recall that the standard contact structure on $S^{3}$ is the 2-dimensional distribution $\xi_{\text {st }} \subset T S^{3}$ given by the complex tangents, where $S^{3}$ is viewed as the boundary of the unit 4 -ball in $\mathbb{C}^{2}$. We say that a knot in $S^{3}$ is Legendrian if it is everywhere tangent to $\xi_{\text {st }}$. To every Legendrian knot $L \subset S^{3}$ one can associate its Thurston-Bennequin number $\operatorname{tb}(L) \in \mathbb{Z}$, which is invariant under Legendrian isotopies of $L$ [1. Given a knot $K \subset S^{3}$, let $\operatorname{TB}(K)$ denote the maximal Thurston-Bennequin number of $K$, defined as 
$\operatorname{TB}(K)=\max \{t b(L) \mid L$ is Legendrian and smoothly isotopic to $K\}$.

Let $g_{s}(K)$ denote the slice genus (aka the 4 -ball genus) of $K$. Let $S_{r}^{3}(K)$ be the oriented 3-manifold given by rational $r$-surgery on a knot $K \subset S^{3}$.

Theorem 1.1 Let $K \subset S^{3}$ be a knot such that

$$
g_{s}(K)>0 \quad \text { and } \quad \operatorname{TB}(K)=2 g_{s}(K)-1 .
$$

Then, the oriented 3-manifold $S_{r}^{3}(K)$ carries positive, tight contact structures for every $r \neq 2 g_{s}(K)-1$.

Remark By the slice Bennequin inequality 33, for any knot $K \subset S^{3}$ we have

$$
\mathrm{TB}(K) \leq 2 g_{s}(K)-1 \text {. }
$$

Moreover, by [2, 3] (see 11, page 123]), if $K$ is an algebraic knot then

$$
\mathrm{TB}(K)=2 g(K)-1,
$$

where $g(K)$ is the Seifert genus of $K$. Since $g_{s}(K) \leq g(K)$, it follows that the family of knots $K$ satisfying the assumption of Theorem 1.1 contains all nontrivial algebraic knots. In fact, there are non-fibered, hence non-algebraic, knots satisfying the same assumption, as for example certain negative twist knots 1

Let $T \subset S^{3}$ be the right-handed trefoil. Since $T$ is algebraic, Theorem 1.1 applies. In particular, since $S_{2}^{3}(T)=-\Sigma(2,3,4)$ and $S_{3}^{3}(T)=-\Sigma(2,3,3)$, Theorem 1.1 immediately implies the following result, which solves a wellknown open problem [11, Question 8]:

Corollary 1.2 The Brieskorn spheres $-\Sigma(2,3,3)$ and $-\Sigma(2,3,4)$ carry positive, tight contact structures.

\footnotetext{
${ }^{1}$ Let $K_{q}$ be a twist knot with $q<0$ twists (cf [32, page 112]). It is easy to find a Legendrian representative of $K_{q}$ with Thurston-Bennequin number equal to 1 . On the other hand, by resolving the clasp it follows that $g_{s}\left(K_{q}\right) \leq 1$. Therefore the slice Bennequin inequality implies $g_{s}\left(K_{q}\right)=T B\left(K_{q}\right)=1$. The knots $K_{q}$ are not fibered for $q<-1$ because the leading coefficient of their Alexander polynomial is not equal to 1 .
} 
Remarks (1) The proof of Theorem 1.1 shows that Figures 1 and 2 below provide explicit descriptions of the tight contact structures of Corollary [1.2

(2) Theorem 1.1 is optimal for the right-handed trefoil knot $T=T_{3,2}$, because $S_{1}^{3}(T)=-\Sigma(2,3,5)$ is known not to carry positive, tight contact structures [12]. On the other hand, it is natural to ask whether the same is true for other torus knots. We address this question in the companion paper [22].

Recall that a symplectic filling of a contact three-manifold $(Y, \xi)$ is a pair $(X, \omega)$ consisting of a smooth, compact, connected four-manifold $X$ and a symplectic form $\omega$ on $X$ such that, if $X$ is oriented by $\omega \wedge \omega, \partial X$ is given the boundary orientation and $Y$ is oriented by $\xi$, then $\partial X=Y$ and $\left.\omega\right|_{\xi} \neq 0$ at every point of $\partial X$. As an application of Theorem 1.1 we prove the following result, which should be compared with the results of [20, 21].

Theorem 1.3 For each $m \in \mathbb{N}$ there is a Seifert fibered rational homology sphere $M_{m}$ carrying at least $m$ pairwise non-isomorphic tight, not symplectically fillable contact structures.

The paper is organized as follows. In Section 2 we describe the necessary background in contact surgery and Heegaard Floer theory. In Sections 3 and 4 we prove, respectively, Theorems 1.1 and 1.3 .

Acknowledgements The first author was partially supported by MURST, and he is a member of EDGE, Research Training Network HPRN-CT-200000101, supported by The European Human Potential Programme. The second author would like to thank Peter Ozsváth and Zoltán Szabó for many useful discussions regarding their joint work. The second author was partially supported by OTKA T34885.

\section{Surgeries and Ozsváth-Szabó invariants}

\section{Contact surgery}

Let $(Y, \xi)$ be a contact 3 -manifold. The framing of a Legendrian knot $K \subset Y$ naturally induced by $\xi$ is called the contact framing of $K$. Given a Legendrian knot $K$ in a contact 3-manifold $(Y, \xi)$ and a non-zero rational number $r \in \mathbb{Q}$, one can perform contact $r$-surgery along $K$ to obtain a new contact 3 -manifold $\left(Y^{\prime}, \xi^{\prime}\right)$ [4, 5]. Here $Y^{\prime}$ is the 3 -manifold obtained by smooth $r$-surgery along 
$K$ with respect to the contact framing, while $\xi^{\prime}$ is constructed by extending $\xi$ from the complement of a standard neighborhood of $K$ to a tight contact structure on the glued-up solid torus. If $r \neq 0$ such an extension always exists, and for $r=\frac{1}{k} \quad(k \in \mathbb{Z})$ it is unique [15]. When $r=-1$ the corresponding contact surgery coincides with Legendrian surgery along $K$ [9, 14, 34.

As an illustration of the contact surgery construction, consider the Legendrian trefoil knot $\mathcal{T}$ represented by the Legendrian front (see eg 14 for notation) of Figure 1, Since the coefficient +1 represents the contact surgery coefficient and $\operatorname{tb}(\mathcal{T})=1$, the picture represents a contact structure on the oriented 3manifold obtained by a smooth $(+2)$-surgery on a right-handed trefoil knot, that is on $-\Sigma(2,3,4)$.

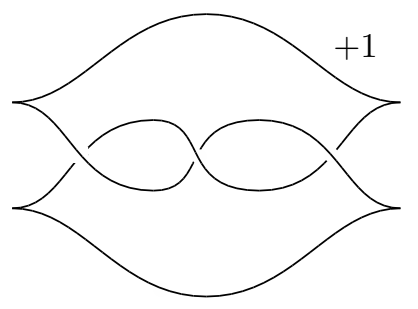

Figure 1: $\quad$ A contact structure on $-\Sigma(2,3,4)$

According to [5, Proposition 7], a contact $r=\frac{p}{q}$-surgery $(p, q \in \mathbb{N})$ on a Legendrian knot $K$ is equivalent to a contact $\frac{1}{k}$-surgery on $K$ followed by a contact $\frac{p}{q-k p}$-surgery on a Legendrian pushoff of $K$ for any integer $k \in \mathbb{N}$ such that $q-k p<0$. Moreover, by [5, Proposition 3] any contact $r$-surgery along $K \subset(Y, \xi)$ with $r<0$ is equivalent to a Legendrian surgery along a Legendrian link $\mathbb{L}=\cup_{i=0}^{m} L_{i}$ which is determined via a simple algorithm by the Legendrian knot $K$ and the contact surgery coefficient $r$. The algorithm to obtain $\mathbb{L}$ is the following. Let

$$
\left[a_{0}, \ldots, a_{m}\right], \quad a_{0}, \ldots a_{m} \geq 2
$$

be the continued fraction expansion of $1-r$. To obtain the first component $L_{0}$, push off $K$ using the contact framing and stabilize it $a_{0}-2$ times. Then, push off $L_{0}$ and stabilize it $a_{1}-2$ times. Repeat the above scheme for each of the remaining pivots of the continued fraction expansion. Since there are $a_{i}-1$ inequivalent ways to stabilize a Legendrian knot $a_{i}-2$ times, this construction yields $\Pi_{i=0}^{m}\left(a_{i}-1\right)$ potentially different contact structures.

For example, according to the algorithm just described, any contact $(+2)-$ surgery on $\mathcal{T}$ is equivalent to one of the contact surgeries of Figure 2 (the coefficients indicate surgery with respect to the contact framings). 

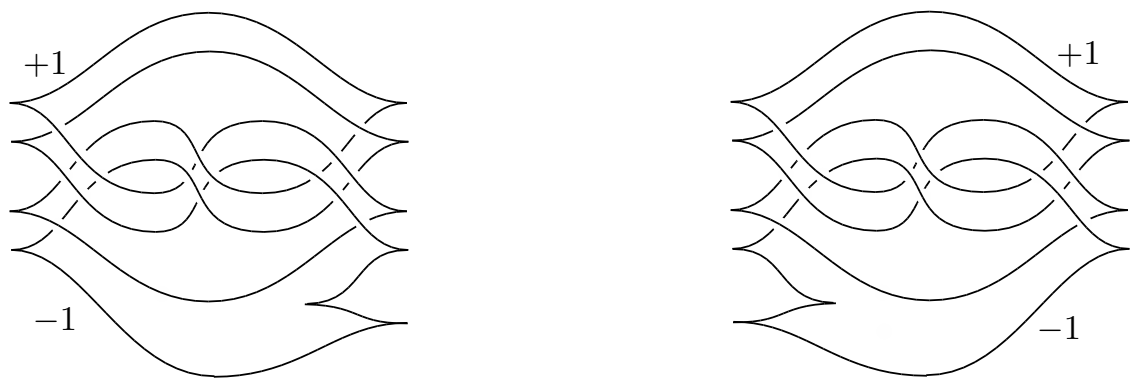

Figure 2: $\quad$ Contact structures on $-\Sigma(2,3,3)$

Since, by [4. Proposition 9], a contact $\frac{1}{k}$-surgery $(k \in \mathbb{N})$ on a Legendrian knot $K$ can be replaced by $k$ contact $(+1)$-surgeries on $k$ Legendrian pushoffs of $K$, it follows that any contact rational $r$-surgery $(r \neq 0)$ can be replaced by contact $( \pm 1)$-surgery along a Legendrian link; for a related discussion see also [6, 21.

\section{The Ozsváth-Szabó invariants of 3-manifolds}

The Ozsváth-Szabó invariants [24, 25, 26] assign to each oriented $\operatorname{spin}^{c} 3$ manifold $(Y, \mathbf{s})$ a finitely generated Abelian group $\widehat{H F}(Y, \mathbf{s})$, and to each oriented $\operatorname{spin}^{c}$ cobordism $(W, \mathbf{t})$ between $\left(Y_{1}, \mathbf{s}_{1}\right)$ and $\left(Y_{2}, \mathbf{s}_{2}\right)$ a homomorphism

$$
F_{W, \mathbf{t}}: \widehat{H F}\left(Y_{1}, \mathbf{s}_{1}\right) \rightarrow \widehat{H F}\left(Y_{2}, \mathbf{s}_{2}\right) \text {. }
$$

For simplicity, in the following we will use these homology theories with $\mathbb{Z} / 2 \mathbb{Z}$ coefficients. In this setting, $\widehat{H F}(Y, \mathbf{s})$ is a finite dimensional vector space over the field $\mathbb{Z} / 2 \mathbb{Z}$. Define

$$
\widehat{H F}(Y)=\bigoplus_{\mathbf{s} \in \operatorname{Spin}^{c}(Y)} \widehat{H F}(Y, \mathbf{s})
$$

Since there are only finitely many $\operatorname{spin}^{c}$ structures with nonvanishing invariants [25. Theorem 7.1], $\widehat{H F}(Y)$ is still finite dimensional.

An important ingredient of our proofs is the following result, which appears implicitly in the papers of Ozsváth and Szabó (see especially [30]). We provide a detailed proof for completeness.

Proposition 2.1 Let $W$ be a cobordism obtained by attaching a 2-handle to a 3-manifold $Y$ with $b_{1}(Y)=0$. Let $\mathbf{t}_{0} \in \operatorname{Spin}^{c}(W)$, and suppose that $W$ 
contains a smoothly embedded, closed, oriented surface $\Sigma$ of genus $g(\Sigma)>0$ such that

$$
\Sigma \cdot \Sigma \geq 0 \quad \text { and } \quad\left|\left\langle c_{1}\left(\mathbf{t}_{0}\right),[\Sigma]\right\rangle\right|+\Sigma \cdot \Sigma>2 g(\Sigma)-2 .
$$

Then, $F_{W, \mathbf{t}_{0}}=0$.

Proof Arguing by contradiction, suppose that $F_{W, \mathbf{t}_{0}} \neq 0$. By a fundamental property of the invariants [26] there are only finitely many $\operatorname{spin}^{c}$ structures $\mathbf{t}_{1}, \ldots, \mathbf{t}_{k} \in \operatorname{Spin}^{c}(W)$ such that $F_{W, \mathbf{t}_{i}} \neq 0$. Moreover, by [26, Theorem 3.6] we have

$$
F_{W, \mathbf{t}_{0}} \neq 0 \quad \Longleftrightarrow \quad F_{W, \overline{\mathbf{t}}_{0}} \neq 0
$$

where $\overline{\mathbf{t}}_{0}$ is the $\operatorname{spin}^{c}$ structure conjugate to $\mathbf{t}_{0}$. Therefore, up to replacing $\mathbf{t}_{0}$ with one of the $\mathbf{t}_{i}$ 's we may assume that

$$
\left\langle c_{1}\left(\mathbf{t}_{0}\right),[\Sigma]\right\rangle=\left|\left\langle c_{1}\left(\mathbf{t}_{0}\right),[\Sigma]\right\rangle\right|=\max \left\{\left\langle c_{1}\left(\mathbf{t}_{i}\right),[\Sigma]\right\rangle \mid i=1, \ldots, k\right\} .
$$

Let $\Sigma \cdot \Sigma=n$, and let $\widehat{W}$ be the smooth $4-$ manifold obtained by blowing up $W$ at $n$ distinct points of $W \backslash \Sigma$. Choose exceptional classes

$$
e_{1}, \ldots, e_{n} \in H_{2}(\widehat{W})
$$

and let $\widehat{\mathbf{t}}_{0}$ denote the unique $\operatorname{spin}^{c}$ structure on $\widehat{W}$ such that $\left.\widehat{\mathbf{t}}_{0}\right|_{W}=\mathbf{t}$ and $\left\langle c_{1}\left(\widehat{\mathbf{t}}_{0}\right), e_{i}\right\rangle=1$ for every $i=1, \ldots, n$.

Let $\widehat{\Sigma} \subset \widehat{W}$ be a smooth, oriented surface obtained by piping $\Sigma$ to the $n$ exceptional spheres, so that

$$
[\widehat{\Sigma}]=[\Sigma]+\sum_{i=1}^{n} e_{i}
$$

Let $\gamma \subset \widehat{W}$ be a properly embedded arc (disjoint from $Y$ and $\widehat{\Sigma}$ away from its endpoints) connecting $Y$ to $\widehat{\Sigma}$. Denote by $\widehat{W}_{1}$ a closed regular neighborhood of the union $Y \cup \gamma \cup \widehat{\Sigma}$, and let $\widehat{W}_{2}$ be the closure of $\widehat{W} \backslash \widehat{W}_{1}$.

Let

$$
\mathcal{S}=\left\{\widehat{\mathbf{t}} \in \operatorname{Spin}^{c}(\widehat{W}) \quad|\widehat{\mathbf{t}}|_{\widehat{W}_{i}}=\left.\widehat{\mathbf{t}}_{0}\right|_{\widehat{W}_{i}}, i=1,2\right\} .
$$

By the composition law [26, Theorem 3.4] we have

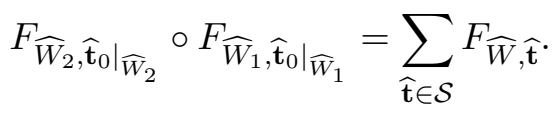

We are going to show that the sum at the right hand side of (2.2) admits at most one nontrivial term. In fact, we shall prove that

$$
\widehat{\mathbf{t}} \in \mathcal{S} \text { and } F_{\widehat{W}, \widehat{\mathbf{t}}} \neq 0 \Longrightarrow \widehat{\mathbf{t}}=\widehat{\mathbf{t}}_{0}
$$


Recall that $\operatorname{Spin}^{c}(\widehat{W})$ admits a free and transitive action of $H^{2}(\widehat{W} ; \mathbb{Z})$. Hence, there is an element $L \in H^{2}(\widehat{W} ; \mathbb{Z})$ such that

$$
\widehat{\mathbf{t}}-\widehat{\mathbf{t}}_{0}=L \text {. }
$$

Since

$$
\widehat{\mathbf{t}}_{\widehat{W}_{i}}=\left.\widehat{\mathbf{t}}_{0}\right|_{\widehat{W}_{i}}, \quad i=1,2,
$$

we have, in particular, $\left.L\right|_{Y}=0$. Therefore $L$ is the image of an element $A \in H^{2}(\widehat{W}, Y ; \mathbb{Z})$ under the restriction map $H^{2}(\widehat{W}, Y ; \mathbb{Z}) \rightarrow H^{2}(\widehat{W} ; \mathbb{Z})$. Our plan is to show that $\widehat{\mathbf{t}}=\widehat{\mathbf{t}}_{0}$ by proving that $A=0$. Since

$$
H_{1}(W, Y ; \mathbb{Z}) \cong H_{1}(\widehat{W}, Y ; \mathbb{Z})=0,
$$

the universal coefficient theorem implies that

$$
H^{2}(\widehat{W}, Y ; \mathbb{Z}) \cong \operatorname{Hom}\left(H_{2}(\widehat{W}, Y ; \mathbb{Z}), \mathbb{Z}\right),
$$

therefore to show $A=0$ it is enough to show $2 A=0$, and $2 A$ is determined by its values on the elements of $H_{2}(\widehat{W}, Y ; \mathbb{Z})$. But since $b_{1}(Y)=0$, it suffices to show that $2 A$ evaluates trivially on the image of the map

$$
i_{*}: H_{2}(\widehat{W} ; \mathbb{Z}) \longrightarrow H_{2}(\widehat{W}, Y ; \mathbb{Z}) .
$$

On the other hand, since $\widehat{\Sigma} \subset \widehat{W}_{1}$, if $\widehat{\mathbf{t}} \in \mathcal{S}$ then $\left\langle c_{1}(\widehat{\mathbf{t}}),[\widehat{\Sigma}]\right\rangle=\left\langle c_{1}\left(\widehat{\mathbf{t}}_{0}\right),[\widehat{\Sigma}]\right\rangle$, ie,

$$
\left\langle c_{1}\left(\left.\widehat{\mathbf{t}}\right|_{W}\right),[\Sigma]\right\rangle+\sum_{i=1}^{n}\left\langle c_{1}(\widehat{\mathbf{t}}), e_{i}\right\rangle=\left\langle c_{1}\left(\mathbf{t}_{0}\right),[\Sigma]\right\rangle+n .
$$

Moreover, by the blow-up formula [26, Theorem 3.7] if $\widehat{\mathbf{t}} \in \operatorname{Spin}^{c}(\widehat{W})$ then

$$
F_{W,\left.\mathbf{t}\right|_{W}} \neq 0 \quad \Longleftrightarrow \quad F_{\widehat{W}, \widehat{\mathbf{t}}} \neq 0 \quad \Longrightarrow \quad\left|\left\langle c_{1}(\widehat{\mathbf{t}}), e_{i}\right\rangle\right|=1, \quad i=1, \ldots, n .
$$

Therefore, if $F_{\widehat{W}, \widehat{t}} \neq 0$, by Equations (2.1) and (2.3) we have

$$
\left\langle c_{1}\left(\left.\widehat{\mathbf{t}}\right|_{W}\right),[\Sigma]\right\rangle=\left\langle c_{1}\left(\mathbf{t}_{0}\right),[\Sigma]\right\rangle \quad \text { and } \quad\left\langle c_{1}(\widehat{\mathbf{t}}), e_{i}\right\rangle=\left\langle c_{1}\left(\widehat{\mathbf{t}}_{0}\right), e_{i}\right\rangle=1, \quad i=1, \ldots, n \text {. }
$$

It follows that $c_{1}(\widehat{\mathbf{t}})=c_{1}\left(\widehat{\mathbf{t}}_{0}\right)$. Therefore, for every $\alpha \in H_{2}(\widehat{W} ; \mathbb{Z})$ we have

$$
\left\langle 2 A, i_{*}(\alpha)\right\rangle=\langle 2 L, \alpha\rangle=\left\langle c_{1}(\widehat{\mathbf{t}})-c_{1}\left(\widehat{\mathbf{t}}_{0}\right), \alpha\right\rangle=0 .
$$

Thus, $\widehat{\mathbf{t}}=\widehat{\mathbf{t}}_{0}$, and the right-hand side of Equation (2.2) reduces to $F_{\widehat{W}, \widehat{\mathbf{t}}_{0}}$.

Now observe that $\widehat{W}_{1}$ is a cobordism from $Y$ to $Y \# S^{1} \times \widehat{\Sigma}$, and since

$$
\left\langle c_{1}\left(\widehat{\mathbf{t}}_{0}\right),[\widehat{\Sigma}]\right\rangle=\left\langle c_{1}\left(\mathbf{t}_{0}\right),[\Sigma]\right\rangle+n>2 g(\widehat{\Sigma})-2,
$$

by the adjunction inequality [25, Theorem 7.1] the group

$$
\widehat{H F}\left(Y \# S^{1} \times \widehat{\Sigma},\left.\widetilde{\mathbf{t}}_{0}\right|_{S^{1} \times \widehat{\Sigma}}\right)
$$

is trivial. But this group is the domain of the map $F_{\widehat{W}_{2},\left.\widehat{\mathbf{t}}_{0}\right|_{\widehat{W}_{2}}}$. Thus, Equation (2.2) implies that $F_{\widehat{W}, \widehat{t}_{0}}=0$ and therefore $F_{W, \mathbf{t}_{0}}=0$, which gives the desired contradiction. 


\section{Contact Ozsváth-Szabó invariants}

In 28] Ozsváth and Szabó defined an invariant

$$
c(Y, \xi) \in \widehat{H F}\left(-Y, \mathbf{s}_{\xi}\right) /\langle \pm 1\rangle
$$

for a contact $3-$ manifold $(Y, \xi)$, where $\mathbf{s}_{\xi}$ denotes the $\operatorname{spin}^{c}$ structure induced by the contact structure $\xi$. Since in this paper we are using this homology theory with $\mathbb{Z} / 2 \mathbb{Z}$ coefficients, the above sign ambiguity for $c(Y, \xi)$ does not occur. It is proved in 28] that if $(Y, \xi)$ is overtwisted then $c(Y, \xi)=0$, and if $(Y, \xi)$ is Stein fillable then $c(Y, \xi) \neq 0$. In particular, $c\left(S^{3}, \xi_{\mathrm{st}}\right) \neq 0$. We are going to use the properties of $c(Y, \xi)$ described in the following theorem and corollary.

Theorem 2.2 (21], Theorem 2.3) Suppose that $\left(Y^{\prime}, \xi^{\prime}\right)$ is obtained from $(Y, \xi)$ by a contact $(+1)$-surgery. Let $-X$ be the cobordism induced by the surgery with reversed orientation. Define

$$
F_{-X}:=\sum_{\mathbf{t} \in \operatorname{Spin}^{c}(-X)} F_{-X, \mathbf{t}} .
$$

Then,

$$
F_{-X}(c(Y, \xi))=c\left(Y^{\prime}, \xi^{\prime}\right) .
$$

In particular, if $c\left(Y^{\prime}, \xi^{\prime}\right) \neq 0$ then $(Y, \xi)$ is tight.

Corollary 2.3 (21], Corollary 2.4) If $c\left(Y_{1}, \xi_{1}\right) \neq 0$ and $\left(Y_{2}, \xi_{2}\right)$ is obtained from $\left(Y_{1}, \xi_{1}\right)$ by Legendrian surgery along a Legendrian knot, then $c\left(Y_{2}, \xi_{2}\right) \neq 0$. In particular, $\left(Y_{2}, \xi_{2}\right)$ is tight.

\section{The surgery exact triangle}

Here we describe what is usually called the surgery exact triangle for the Ozsváth-Szabó homologies.

Let $Y$ be a closed, oriented 3-manifold and let $K \subset Y$ be a framed knot with framing $f$. Let $Y(K)$ denote the 3 -manifold given by surgery along $K \subset Y$ with respect to the framing $f$. The surgery can be viewed at the 4 -manifold level as a 4-dimensional 2-handle addition. The resulting cobordism $X$ induces a homomorphism

$$
F_{X}:=\sum_{\mathbf{t} \in \operatorname{Spin}^{c}(X)} F_{X, \mathbf{t}}: \widehat{H F}(Y) \rightarrow \widehat{H F}(Y(K))
$$


obtained by summing over all $\operatorname{spin}^{c}$ structures on $X$. Similarly, there is a cobordism $U$ defined by adding a 2-handle to $Y(K)$ along a normal circle $N$ to $K$ with framing -1 with respect to a normal disk to $K$. The boundary components of $U$ are $Y(K)$ and the 3-manifold $Y^{\prime}(K)$ obtained from $Y$ by a surgery along $K$ with framing $f+1$. As before, $U$ induces a homomorphism

$$
F_{U}: \widehat{H F}(Y(K)) \rightarrow \widehat{H F}\left(Y^{\prime}(K)\right) .
$$

It is proved in 25, Theorem 9.16] that

$$
\operatorname{ker} F_{U}=\operatorname{Im} F_{X} .
$$

The above construction can be repeated starting with $Y(K)$ and $N \subset Y(K)$ equipped with the framing specified above: we get $U$ (playing the role previously played by $X$ ) and a new cobordism $V$ starting from $Y^{\prime}(K)$, given by attaching a 4-dimensional 2-handle along a normal circle $C$ to $N$ with framing -1 with respect to a normal disk. It is easy to check that this last operation yields $Y$ at the 3-manifold level. Again, we have $\operatorname{ker} F_{V}=\operatorname{Im} F_{U}$. Moreover, we can apply the construction once again, and denote by $W$ the cobordism obtained by attaching a 2-handle along a normal circle $D$ to $C$ with framing -1 . In fact, $W$ is orientation-preserving diffeomorphic to $X$. This fact is explained in Figure 3. where the first picture represents $W$ and the last picture represents $X$. In the figure, the framed dotted circle is the attaching circle of the 2-handle. The first diffeomorphism in Figure 3 is obtained by "blowing down" the framed knot $C$. In other words, the first two pictures represent 2-handles attached to diffeomorphic 3-manifolds, and show that the corresponding attaching maps commute with the given diffeomorphism. The second diffeomorphism is obtained by a handle slide, and the third diffeomorphism by erasing a cancelling pair. It follows immediately from Equation (2.4) that the homomorphisms $F_{X}, F_{U}$ and $F_{V}$ fit into the surgery exact triangle:

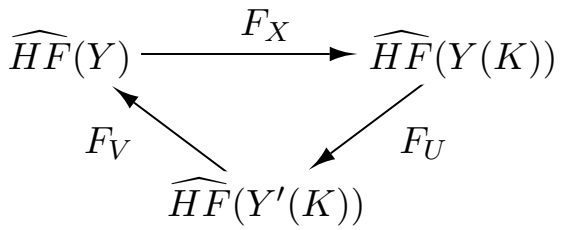

\footnotetext{
${ }^{2}$ In fact, the maps $F_{U}$ and $F_{X}$ were defined in 25] by counting pseudo-holomorphic triangles in a Heegaard triple, but an easy comparison with the maps associated to 2-handles defined in [26. Subsection 4.1] shows that $F_{U}$ and $F_{X}$ are the sums of maps associated to cobordisms given above (see the discussion at the beginning of 27 . Section 3]).
} 


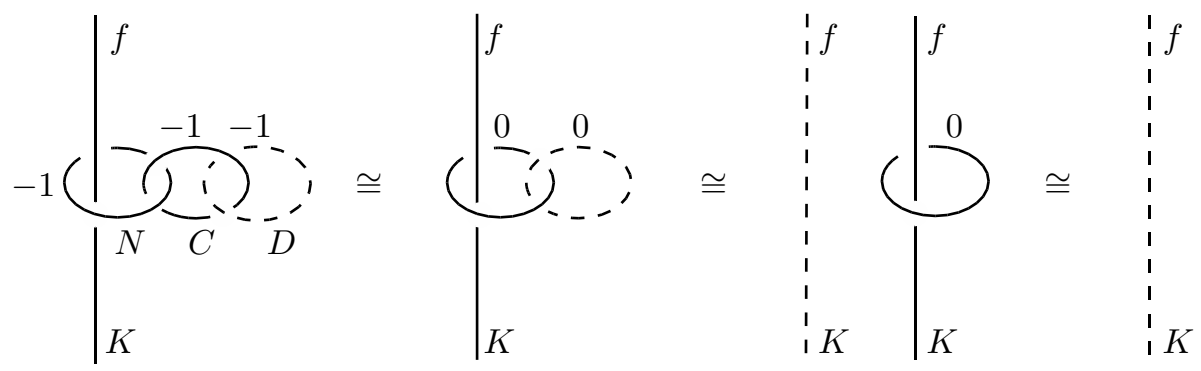

Figure 3: The diffeomorphism between $W$ and $X$

Remark Given an exact triangle of vector spaces and homomorphisms

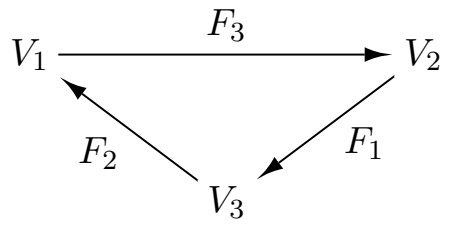

we have

$$
\operatorname{dim} V_{i} \leq \operatorname{dim} V_{j}+\operatorname{dim} V_{k}
$$

for $\{i, j, k\}=\{1,2,3\}$. Moreover, equality holds in (2.6) if and only if $F_{i}=0$.

\section{The proof of Theorem 1.1}

Let $L$ be a Legendrian knot smoothly isotopic to $K$ with

$$
t:=\operatorname{tb}(L)=2 g_{s}(K)-1 .
$$

Let $r \in \mathbb{Q} \backslash\{t\}$ and $r^{\prime}=r-t$. Then, any contact $r^{\prime}$-surgery along $L$ yields a contact structure on $S_{r}^{3}(K)$.

If $r<t=2 g_{s}(K)-1$ then $r^{\prime}<0$. Since any contact $r^{\prime}$-surgery along $L$ can be realized by Legendrian surgery, the resulting contact structure is Stein fillable and hence tight [10. Therefore, to prove Theorem 1.1]it suffices to show that any contact $r^{\prime}$-surgery along $L$ with $r^{\prime}>0$ yields a contact structure on $S_{r}^{3}(K)$ with non-zero contact Ozsváth-Szabó invariant.

Let $\left(Y_{k}, \xi_{k}\right)$, with $k$ any positive integer, denote the result of contact $\frac{1}{k}$-surgery along $L$. If $r^{\prime}>0$, any contact $r^{\prime}$-surgery along $L$ is equivalent to a sequence of Legendrian surgeries on $\left(Y_{k}, \xi_{k}\right)$ for some $k>0$. Therefore, by Corollary 2.3 
it suffices to prove that the contact invariants of $\left(Y_{k}, \xi_{k}\right)$ do not vanish. We claim that, for every $k \geq 1$,

$$
c\left(Y_{k}, \xi_{k}\right) \neq 0 .
$$

We are going to prove the claim by induction on $k$. To start the induction, we examine the case $k=1$ first.

Observe that $Y_{1}(L)=S_{2 g_{s}}^{3}(K)$, and let $-X$ be the cobordism induced by contact $(+1)$-surgery along $L$ with reversed orientation. Then it is easy to check that, according to the discussion preceding (2.5), the homomorphism $F_{-X}$ fits into an exact triangle

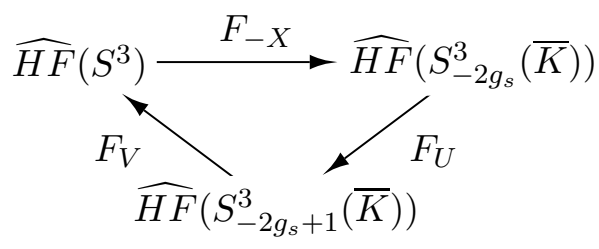

where $\bar{K}$ denotes the mirror image of $K$. By Theorem 2.2 the map $F_{-X}$ sends the non-zero contact Ozsváth-Szabó invariant $c\left(S^{3}, \xi_{s t}\right)$ to $c\left(Y_{1}(L), \xi_{1}\right)$. It is now easy to see that the cobordism $V$ viewed up-side down is obtained by attaching a 2-handle to $S^{3}$ along $K$ with framing $2 g_{s}(K)-1$. Therefore, $V$ contains a smoothly embedded surface of genus $g_{s}(K)$ and self-intersection $2 g_{s}(K)-1$. It follows by Proposition 2.1 that $F_{V}=0$. By exactness this means that $F_{-X}$ is injective, therefore

$$
F_{-X}\left(c\left(S^{3}, \xi_{s t}\right)\right)=c\left(Y_{1}(L), \xi_{1}\right) \neq 0,
$$

and the claim (3.1) is proved for $k=1$. We are left to prove that

$$
c\left(Y_{k}, \xi_{k}\right) \neq 0 \quad \Longrightarrow c\left(Y_{k+1}, \xi_{k+1}\right) \neq 0
$$

for every $k \geq 1$.

By construction, $\left(Y_{k+1}, \xi_{k+1}\right)$ is given as contact $(+1)$-surgery on a Legendrian knot in $\left(Y_{k}, \xi_{k}\right)$. If $X_{k}$ denotes the corresponding cobordism, by Theorem 2.2 we have

$$
F_{-X_{k}}\left(c\left(Y_{k}, \xi_{k}\right)\right)=c\left(Y_{k+1}, \xi_{k+1}\right) .
$$

The homomorphism $F_{-X_{k}}$ fits into the exact triangle

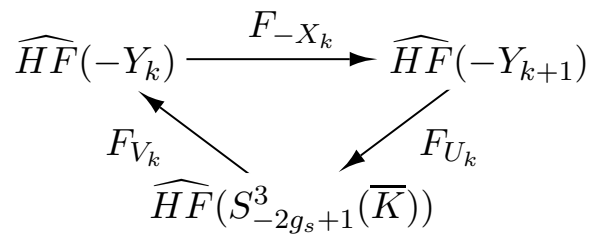


where $\bar{K}$ denotes the mirror image of $K$ and the cobordisms $-X_{k}, U_{k}$ and $V_{k}$ are described in Figure 4 where, in each picture, the framed dashed knot represents the attaching circle of a $2-$ handle giving rise to a cobordism. Remarkably, the third manifold in the triangle is independent of $k$. This is evident from the diffeomorphism given in the lower portion of Figure 4 which is obtained by $k+1$ blowdowns. We are going to show that, for every $k \geq 1$, the cobordism

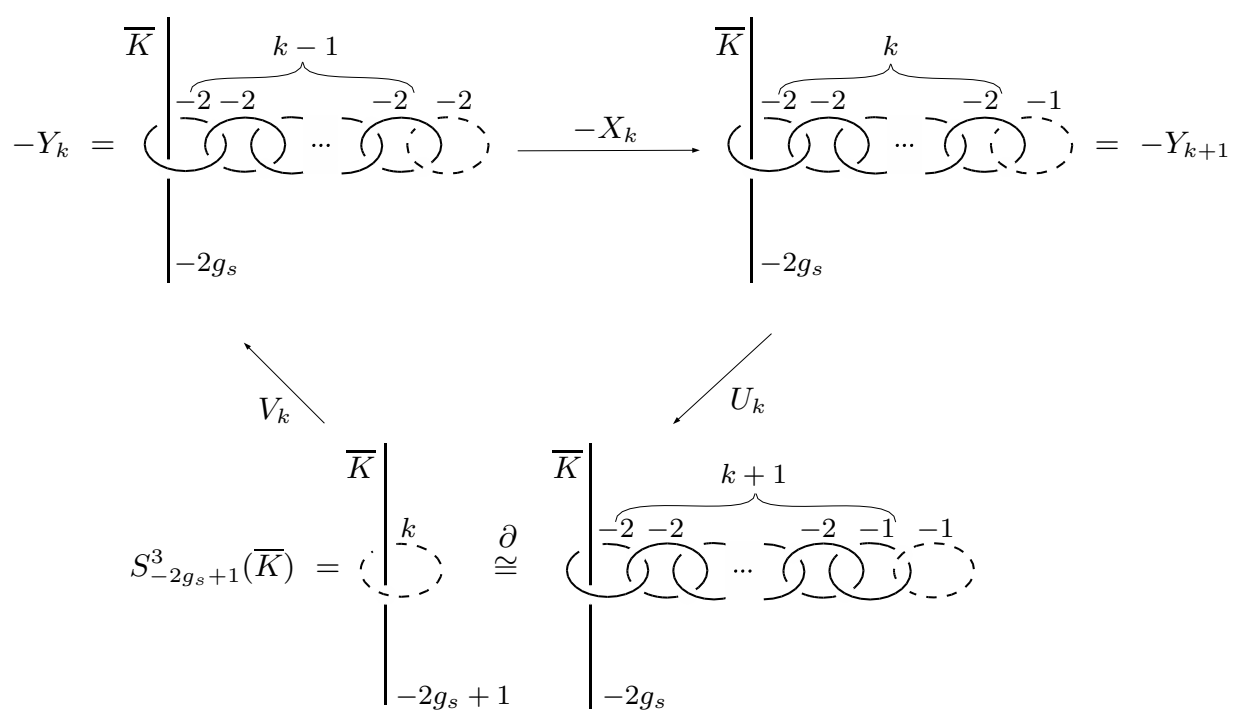

Figure 4: The surgery exact triangle involving $-Y_{k},-Y_{k+1}$ and $S_{-2 g_{s}+1}^{3}(\bar{K})$.

$V_{k}$ contains an embedded surface $\Sigma$ of genus $g(\Sigma)>0$ and

$$
\Sigma \cdot \Sigma \geq 2 g(\Sigma)-1 \text {. }
$$

In view of Proposition 2.1, this implies $F_{V_{k}}=0$, and therefore that $F_{-X_{k}}$ is injective. Assuming $c\left(Y_{k}, \xi_{k}\right) \neq 0$, Equation (3.4) then implies $c\left(Y_{k+1}, \xi_{k+1}\right) \neq$ 0 , and (3.3) follows. Therefore, to finish the proof we only need to establish the existence of the surface $\Sigma \subset V_{k}$ satisfying (3.6).

The cobordism $V_{k}$ is obtained by attaching a 2 -handle to $S_{-t}^{3}(\bar{K})$, where the corresponding framed attaching circle is shown in the lower left portion of Figure 4. We can think of $S_{-t}^{3}(\bar{K})$ as the boundary of the 4 -manifold $Z$ obtained by attaching a 2 -handle $H_{\bar{K}}$ to the 4 -ball along $\bar{K}$ with framing $-t$. Let $W$ denote the union $Z \cup V_{k}$, and let $F \subset Z$ be a smooth surface representing a generator of $H_{2}(Z ; \mathbb{Z})$ obtained by capping off a slicing surface for $\bar{K}$ with the core disk of $H_{\bar{K}}$. Consider a generic pushoff $F^{\prime}$ of $F$, viewed as a surface 
in $W$. When suitably oriented, $F$ and $F^{\prime}$ intersect transversely in $t$ negative points $p_{1}, \ldots, p_{t} \in F^{\prime}$. Consider $t$ generic pushoffs $S_{1}, \ldots, S_{t}$ of the embedded 2 -sphere $S \subset W$ corresponding to the $k$-framed unknot of the lower left portion of Figure 4, oriented so that $S_{i} \cdot F=+1$ for $i=1, \ldots, t$. Each 2-sphere $S_{i}$ intersects $F$ transversely in a unique point $q_{i}$. Consider disjoint, smootly embedded arcs $\gamma_{1}, \ldots, \gamma_{t} \subset F$ such that $\gamma_{i}$ joins $p_{i}$ to $q_{i}$ for each $i=1, \ldots, t$. Let $\nu(F)$ be a small tubular neighborhood of the surface $F$. We can view its boundary $\partial \nu(F)$ as a smooth $S^{1}$ bundle

$$
\pi: \partial \nu(F) \rightarrow F
$$

so that each of the sets $F^{\prime} \cap \partial \nu(F)$ and $\cup_{i=1}^{t} S_{i} \cap \partial \nu(F)$ consists of exactly $t$ fibers of $\pi$. The immersed surface

$$
\widetilde{\Sigma}=F^{\prime} \backslash \nu(F) \cup_{i=1}^{t} \pi^{-1}\left(\gamma_{i}\right) \cup_{i=1}^{t} S_{i} \backslash \nu(F) \subset W
$$

is contained in the complement of $F$. The singularities of $\widetilde{\Sigma}$ come from the intersections among $S_{1}, \ldots, S_{t}$ and $F^{\prime}$. Resolving those singularities one gets a smoothly embedded surface which can be isotoped to a surface $\Sigma \subset V_{k}$. Moreover, a simple computation using the fact that $g\left(F^{\prime}\right)=g_{s}(K)=\frac{1}{2}(t+1)$ shows that

$$
\Sigma \cdot \Sigma=t^{2} k+t \quad \text { and } \quad g(\Sigma)=\frac{t(t-1)}{2} k+\frac{t+1}{2} .
$$

Since

$$
\Sigma \cdot \Sigma-(2 g(\Sigma)-1)=t k>0,
$$

the surface $\Sigma$ satisfies (3.6). This concludes the proof of Theorem 1.1.

\section{The proof of Theorem 1.3}

The following facts (4.1), (4.2) and (4.3) are proved in 25, Propositions 3.1 and 5.1]. Let $L(p, q)$ be a lens space. Then,

$$
\operatorname{dim}_{\mathbb{Z} / 2 \mathbb{Z}} \widehat{H F}(L(p, q))=p .
$$

Let $Y$ be a closed, oriented 3-manifold, and let $-Y$ be the same 3-manifold with reversed orientation. Then,

$$
\widehat{H F}(-Y) \cong \widehat{H F}(Y) .
$$

If $b_{1}(Y)=0$ then

$$
\operatorname{dim}_{\mathbb{Z} / 2 \mathbb{Z}} \widehat{H F}(Y) \geq\left|H_{1}(Y ; \mathbb{Z})\right|
$$


A rational homology $3-$ sphere $Y$ is called an $L$-space if

$$
\operatorname{dim}_{\mathbb{Z} / 2 \mathbb{Z}} \widehat{H F}(Y)=\left|H_{1}(Y ; \mathbb{Z})\right| .
$$

Notice that according to (4.1) lens spaces are $L$-spaces.

Proposition 4.1 Let $K \subset S^{3}$ be a knot such that $g_{s}(K)>0$ and $S_{n}^{3}(K)$ is an $L$-space for some integer $n>0$. Then, $S_{r}^{3}(K)$ is an $L$-space for every rational number $r \geq 2 g_{s}(K)-1$.

Proof The 3 -manifold $S_{r}^{3}(K)$ is an $L$-space for every rational number $r \geq n$. In fact, it follows from [29, Proposition 2.1], that

$$
S_{\frac{a}{b}}^{3}(K) \quad L \text {-space } \Longrightarrow S_{\frac{a+1}{b}}^{3}(K) \quad L \text {-space. }
$$

Suppose $r=\frac{p}{q} \geq n$, and write $p=q n+k$ with $n, k \geq 0$. Then, applying (4.4) $k$ times starting from $S_{n=\frac{p-k}{q}}^{3}(K)$ one deduces that $S_{r}^{3}(K)$ is an $L$-space.

The statement follows immediately if $n<2 g_{s}(K)-1$. If $n \geq 2 g_{s}(K)-1$, it is enough to show that $S_{2 g_{s}(K)-1}^{3}(K)$ is an $L$-space. We do this by backwards induction on $n$. For $n=2 g_{s}(K)-1$ the statement trivially holds. If $n>$ $2 g_{s}(K)-1$, consider the surgery exact triangle given by $S^{3}$ and $K \subset S^{3}$ with framing $n-1$ :

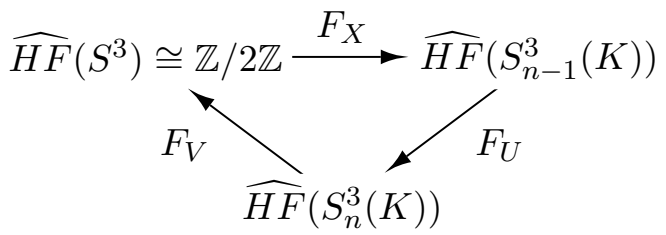

Since the cobordism $X$ contains a smoothly embedded surface $\Sigma$ of genus $g(\Sigma)=g_{s}(K)>0$ and

$$
\Sigma \cdot \Sigma=n-1>2 g_{s}(K)-2,
$$

by Proposition 2.1 we have $F_{X}=0$. This implies that the exact triangle splits, therefore

$$
\widehat{H F}\left(S_{n}^{3}(K)\right) \cong \widehat{H F}\left(S_{n-1}^{3}(K)\right) \oplus \mathbb{Z} / 2 \mathbb{Z} .
$$

Hence, if $S_{n}^{3}(K)$ is an $L$-space then so is $S_{n-1}^{3}(K)$ once $n>2 g_{s}(K)-1$, proving the inductive step.

The following theorem generalizes a result of the first author [18]: Recall that $T_{p, q}$ denotes the positive torus knot of type $(p, q)$. 
Theorem 4.2 For each rational number $r \in[2 n-1,4 n) \cap \mathbb{Q}$, the 3-manifold

$$
S_{r}^{3}\left(T_{2 n+1,2}\right)
$$

carries no fillable contact structures.

Proof Figure 5 describes a 6-step sequence of 3-dimensional Kirby moves which show that the oriented 3 -manifold $S_{r}^{3}\left(T_{2 n+1,2}\right)$ is the boundary of the 4-dimensional plumbing $X$ described by the last picture. The first step of the

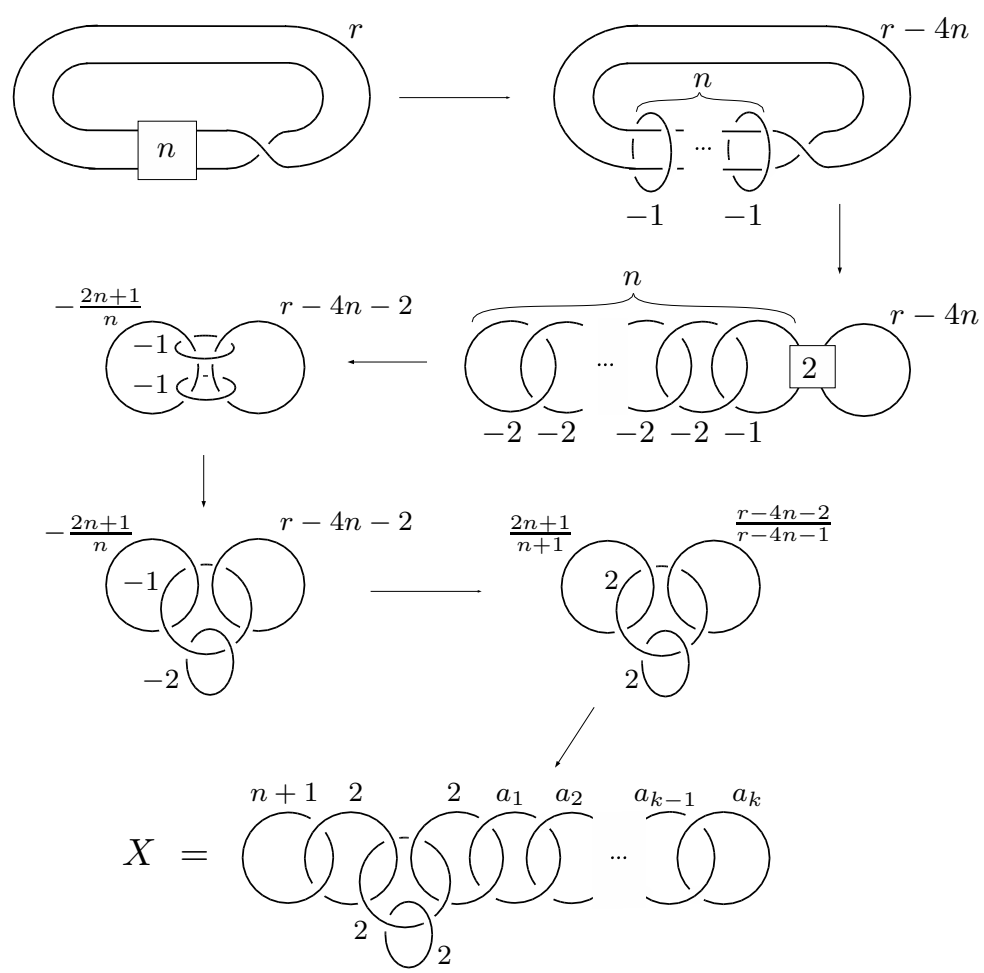

Figure 5: Presentation of $S_{r}^{3}\left(T_{2 n+1,2}\right)$ as boundary of a plumbing

sequence is obtained by $n$ blowups. The second step by $n-1$ handle slides and the third one by two blowups plus a conversion from integer to rational surgery. The fourth step is given by a handle slide, the fifth one by three Rolfsen twists and the sixth one by a conversion from rational to integer surgery. Observe that

$$
1<\frac{r-4 n-2}{r-4 n-1}<2
$$


because $r<4 n$. The coefficients $a_{1}, \ldots, a_{k}$ are given by

$$
\frac{r-4 n-2}{r-4 n-1}=2-\frac{1}{a_{1}-\frac{1}{\ddots \cdot-\frac{1}{a_{k}}}}, \quad a_{1}, \ldots, a_{k} \geq 2 .
$$

By using [17, Theorem 5.2], it is easy to check that the 4-dimensional plumbing $X$ is positive definite. Moreover, the intersection lattice of the plumbing with reversed orientation $-X$ contains the intersection lattice $\Lambda_{a_{1}, n}$ described in Figure 6.

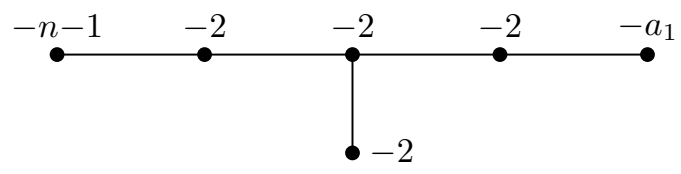

Figure 6: The intersection lattice $\Lambda_{a_{1}, n}$

By [31, Theorem 1.4], every symplectic filling $(W, \omega)$ of a contact 3-manifold $(Y, \xi)$ such that $Y$ is an $L$-space satisfies $b_{2}^{+}(W)=0$. Since $S_{4 n+1}^{3}\left(T_{2 n+1,2}\right)$ is a lens space [23] and, by [16], $2 g_{s}\left(T_{2 n+1,2}\right)-1=2 n-1$, Proposition 4.1 implies that $S_{r}^{3}\left(T_{2 n+1,2}\right)$ is an $L$-space for every $r \geq 2 n-1$. Therefore, every symplectic filling of a contact 3 -manifold of the form $\left(S_{r}^{3}\left(T_{2 n+1,2}\right), \xi\right)$ with $r \geq 2 n-1$ satisfies $b_{2}^{+}(W)=0$.

If $r \in[2 n-1,4 n)$, since $Y=S_{r}^{3}\left(T_{2 n+1,2}\right)$ is a rational homology sphere we can build a negative definite closed 4 -manifold

$$
Z=W \cup_{Y}(-X)
$$

which, according to Donaldson's celebrated theorem [7, 8], must have intersection form $Q_{Z}$ diagonalizable over $\mathbb{Z}$. Since the intersection form $Q_{-X}$ embeds in $Q_{Z}$ it follows that $\Lambda_{a_{1}, n}$ must embed in $Q_{Z}$ as well. But we claim that $\Lambda_{a_{1}, n}$ does not admit an isometric embedding in the diagonal lattice $\mathbb{D}_{m}=\oplus_{m}\langle-1\rangle$. This contradiction forbids the existence of the symplectic filling $W$.

To prove the claim, we argue as in [19, Lemma 3.2]. Suppose there is an isometric embedding $\varphi$ of $\Lambda_{a_{1}, n}$ into $\mathbb{D}_{m}$. Let $e_{1}, \ldots, e_{k}$ be generators of $\mathbb{D}_{m}$ with self-intersection -1 . It is easy to check that, up to composing $\varphi$ with an automorphism of $\mathbb{D}_{m}$, the four generators of $\Lambda_{a_{1}, n}$ corresponding to the vertices of weight $(-2)$ are sent to $e_{1}-e_{2}, e_{2}-e_{3}, e_{3}-e_{4}$ and $e_{3}+e_{4}$. Up to composing 
$\varphi$ with the automorphism of $\mathbb{D}_{m}$ which sends $e_{4}$ to $-e_{4}$ and fixes the remaining ones, the image $v$ of one of the two remaining generators of $\Lambda_{a_{1}, n}$ satisfies

$$
v \cdot\left(e_{3}-e_{4}\right)=0, \quad v \cdot\left(e_{3}+e_{4}\right)=1,
$$

which is impossible because $\left(e_{3}+e_{4}\right)-\left(e_{3}-e_{4}\right)=2 e_{4}$.

Remark The statement of Theorem 4.2 is optimal, in the sense that if $r \notin$ $[2 n-1,4 n)$, then the $3-$ manifold

$$
Y_{n, r}:=S_{r}^{3}\left(T_{2 n+1,2}\right)
$$

supports fillable contact structures. If $r<2 n-1$ then, as observed in the proof of Theorem 1.1 $Y_{n, r}$ carries Stein fillable contact structures. The same holds for $r \geq 4 n$. In fact, examples of Stein fillable contact structures on $Y_{n, r}$ are given by the contact surgery picture of Figure 7 (here we are using our notation as well as the notation of [14]).

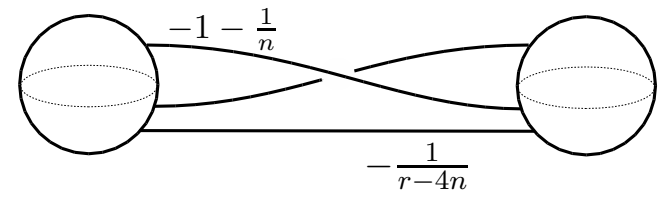

Figure 7: Stein fillable contact structures on $Y_{n, r}$ with $r \geq 4 n$

Proof of Theorem 1.3 Let $m \in \mathbb{N}$, and let $p_{1}, \ldots, p_{m} \in \mathbb{N}$ be consecutive odd primes with either $p_{1}=3$ or $p_{1}=5$, where the choice is made so that

$$
p_{1} \cdots p_{m}=4 k+3
$$

for some $k \in \mathbb{N}$. Now let $\alpha=2 k$, and consider the contact structures obtained via the contact surgeries of Figure 8 .

The underlying $3-$ manifold is

$$
N_{\alpha}:=S_{2+\frac{1}{1+\alpha}}^{3}\left(T_{3,2}\right) .
$$

A simple calculation shows that

$$
H_{1}\left(N_{\alpha} ; \mathbb{Z}\right) \cong \mathbb{Z} /(2 \alpha+3) \mathbb{Z},
$$

with generator the class of the dotted circle $\mu$ drawn in Figure 8 . The possible choices involved in the contact surgery construction, ie, the choices of the $\alpha-1$ stabilizations of the Legendrian $(-\alpha)$-framed unknot, yield contact structures 


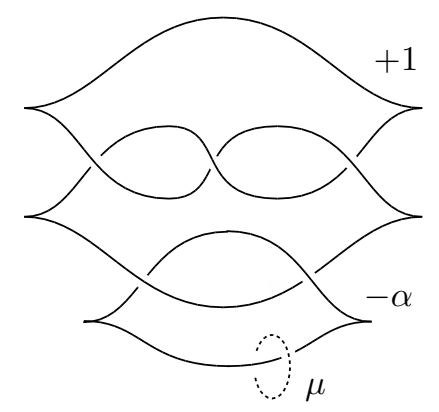

Figure 8: Tight, not fillable contact structures on $N_{\alpha}$

$\xi_{i}(\alpha), i=0, \ldots, \alpha-1$, where $i$ denotes the number of right zig-zags added by the stabilizations. After fixing a suitable orientation for the knots, this implies that

$$
c_{1}\left(\xi_{i}(\alpha)\right)=(2 i-(\alpha-1)) \operatorname{PD}([\mu]) .
$$

(For computations of homotopic data of contact structures defined by surgery diagrams see 6].) Notice that the contact structures $\xi_{i}(\alpha)$ are tight because, since $-\alpha<0$, they are obtained by Legendrian surgeries on the contact structure of Figure 11 which was shown to have non-zero contact Ozsváth-Szabó invariant in the proof of Theorem [1.1. Moreover, since $2+\frac{1}{1+\alpha} \in[1,4)$, by Theorem 1.3 no $\xi_{i}(\alpha)$ is symplectically fillable.

We claim that, for each $j \in\{1, \ldots, m\}$, there exists an index $0 \leq i(j)<\alpha$ such that $c_{1}\left(\xi_{i(j)}(\alpha)\right)$ has order $p_{j}$. Since the primes $p_{j}$ are distinct, the claim implies that the structures $\xi_{i(j)}(\alpha)$ are pairwise non-isomorphic and, since $m$ can be chosen arbitrarily large, it suffices to prove the statement.

To check the claim, define

$$
i(j):=\frac{1}{2}\left(p_{1} \cdots \widehat{p}_{j} \cdots p_{m}+\alpha-1\right) .
$$

Then,

$$
2 i(j)-(\alpha-1)=p_{1} \cdots \widehat{p}_{j} \cdots p_{m}=\frac{1}{p_{j}}(2 \alpha+3),
$$

and therefore $c_{1}\left(\xi_{i(j)}(\alpha)\right)$ has order $p_{j}$. This concludes the proof. 


\section{References}

[1] D Bennequin, Entrelacements et équations de Pfaff, from: "Third Schnepfenried geometry conference, Vol. 1 (Schnepfenried, 1982)", Astérisque 107-108, Soc. Math. France, Paris (1983) 87-161

[2] J Birman, R Williams, Knotted periodic orbits in dynamical systems - I: Lorenz's equations, Topology 22 (1983) 47-82

[3] K Brauner, Klassifikation der singularitäten algebroider Kurven, Abh. Math. Sem. Hamburg 6 (1928) 8-54

[4] F Ding, H Geiges, Symplectic fillability of tight contact structures on torus bundles, Algebr. Geom. Topol. 1 (2001) 153-172

[5] F Ding, H Geiges, A Legendrian surgery presentation of contact 3-manifolds, Math. Proc. Cambridge Philos. Soc. 136 (2004) 583-598

[6] F Ding, H Geiges, A Stipsicz, Surgery diagrams for contact 3-manifolds, Turkish J. Math. 28 (2004) 41-74

[7] S K Donaldson, An application of gauge theory to 4-dimensional topology, J. Differential Geom. 18 (1983) 279-315

[8] S K Donaldson, The Seiberg-Witten equations and 4-manifold topology, Bull. Amer. Math. Soc. 33 (1996) 45-70

[9] Y Eliashberg, Topological characterization of Stein manifolds of dimension $>2$, International J. of Math. 1 (1990) 29-46

[10] Y Eliashberg, Filling by holomorphic discs and its applications, London Math. Soc. Lecture Notes Series 151 (1991) 45-67

[11] J Etnyre, L Ng, Problems in Low Dimensional Contact Topology, Proc. Sympos. Pure Math. 71 (2003) 337-357

[12] J Etnyre, K Honda, On the nonexistence of tight contact structures, Ann. of Math. 153 (2001) 749-766

[13] H Geiges, Contact geometry, from: "Handbook of Differential Geometry vol. 2", (F J E Dillen and L C A Verstraelen, editors), Elsevier, to appear

[14] R Gompf, Handlebody constructions of Stein surfaces, Ann. of Math. 148 (1998) 619-693

[15] K Honda, On the classification of tight contact structures I, Geom. Topol. 4 (2000) 309-368

[16] P Kronheimer, T Mrowka, Gauge theory for embedded surfaces I, Topology 32 (1993) 773-826

[17] W Neumann, F Raymond, Seifert manifolds, plumbing, $\mu$-invariant and orientation reversing maps, from: "Algebraic and geometric topology (Proc. Sympos. Univ. California, Santa Barbara, Calif. 1977)" Lecture Notes in Math. 664, Springer, Berlin (1978) 163-196 
[18] P Lisca, Symplectic fillings and positive scalar curvature, Geom. Topol. 2 (1998) 103-116

[19] P Lisca, On symplectic fillings of 3-manifolds, from: "Proceedings of the $6^{\text {th }}$ Gökova Geometry-Topology Conference", Turkish J. Math. 23 (1999) 151-159.

[20] P Lisca, A Stipsicz, An infinite family of tight, not semi-fillable contact 3manifolds, Geom. Topol. 7 (2003) 1055-1073

[21] P Lisca, A Stipsicz, Seifert fibered contact three-manifolds via surgery, Algebr. Geom. Topol. 4 (2004) 199-217

[22] P Lisca, A Stipsicz, Ozsváth-Szabó invariants and tight contact threemanifolds, II, arXiv:math.SG/0404136

[23] L Moser, Elementary surgery along a torus knot, Pacific J. Math. 38 (1971) 737-745

[24] P Ozsváth, Z Szabó, Holomorphic disks and topological invariants for closed three-manifolds, to appear in Ann. of Math. arXiv:math.SG/0101206

[25] P Ozsváth, Z Szabó, Holomorphic disks and three-manifold invariants: properties and applications, to appear in Ann. of Math. arXiv:math.SG/0105202

[26] P Ozsváth, Z Szabó, Holomorphic triangles and invariants of smooth 4manifolds, to appear in Duke Math. J. arXiv:math.SG/0110169

[27] P. Ozsváth, Z. Szabó, Absolutely graded Floer homologies and intersection forms for four-manifolds with boundary, Adv. Math. 173 (2003) 179-261

[28] P Ozsváth, Z Szabó, Heegaard Floer homologies and contact structures, arXiv:math.SG/0210127

[29] P Ozsváth, Z Szabó, On knot Floer homology and lens space surgery, arXiv:math.GT/0303017

[30] P Ozsváth, Z Szabó, Knot Floer homology and the four-ball genus, Geom. Topol. 7 (2003) 615-639

[31] P Ozsváth, Z Szabó, Holomorphic disks and genus bounds, Geom. Topol. 8 (2004) 311-334

[32] D Rolfsen, Knots and links, Mathematics Lecture Series, No. 7. Publish or Perish, Inc., Berkeley, Calif. (1976)

[33] L Rudolph, The slice genus and the Thurston-Bennequin invariant of a knot, Proc. Amer. Math. Soc. 125 (1997) 3049-3050

[34] A Weinstein, Contact surgery and symplectic handlebodies, Hokkaido Mathematical Journal 20 (1991) 241-251 